\title{
Mapping satellite surfaces and atmospheres with ground-based radio interferometry
}

Corresponding Author: Katherine de Kleer, California Institute of Technology Contact: dekleer@caltech.edu; 626-395-4029

Co-authors: Bryan Butler ${ }^{2}$, Martin Cordiner ${ }^{3,4}$, Imke de Pater ${ }^{5}$, Mark Gurwell ${ }^{6}$, Joseph Lazio ${ }^{7}$, Stefanie Milam ${ }^{3}$, Chris Moeckel ${ }^{5}$, Arielle Moullet ${ }^{8}$, Eric Murphy ${ }^{2}$, R. J. Sault ${ }^{9}$, Joshua Tollefson $^{5}$, Samantha Trumbo ${ }^{1}$.

${ }^{1}$ California Institute of Technology, ${ }^{2}$ National Radio Astronomy Observatory, ${ }^{3}$ NASA Goddard Space Flight Center, ${ }^{4}$ Catholic University of America, ${ }^{5}$ University of California, Berkeley, ${ }^{6}$ Center for Astrophysics | Harvard \& Smithsonian, ${ }^{7}$ Jet Propulsion Laboratory - California Institute of Technology, ${ }^{8}$ SOFIA-USRA, ${ }^{9}$ University of Melbourne.

Abstract: Ground-based interferometry at mm-cm wavelengths provides a powerful tool for characterizing satellite surfaces and atmospheres. We present the science enabled by the ALMA (current) and ngVLA (proposed) arrays, including recent results as well as future work in the context of planned and proposed spacecraft missions.

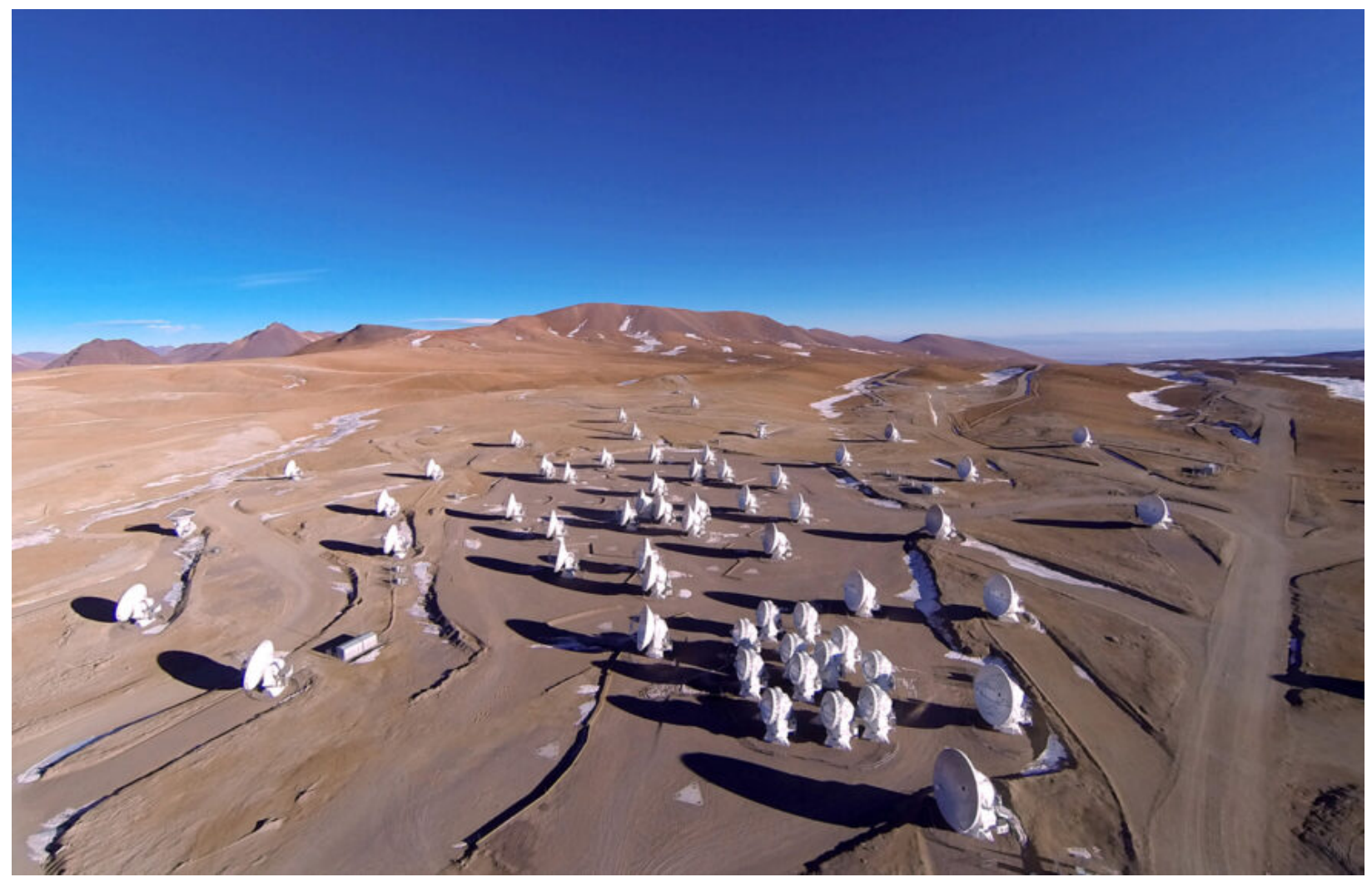

ALMA dishes on the Chajnantor Plateau in Chile (Credit: Ariel Marinkovic / X-Cam) 


\section{Introduction and Context}

The satellites of the giant planets constitute a set of diverse and dynamic worlds, and collectively inform our understanding of the thermal evolution of rocky/icy worlds, the creation and loss of tenuous atmospheres in different bombardment and irradiation environments, and the astrobiological potential of ocean worlds. The next decade is likely to see a transformation of our understanding of the geophysics, chemistry, and biological promise of our population of satellites, due in part to NASA's Europa Clipper and Dragonfly missions and ESA's JUICE mission, as well as Discovery-class missions to Triton (Trident) and Io (IVO) currently under study. Here we describe the role of ground-based radio interferometric observations of the satellites in the next decade, including the context of such studies within the current mission landscape, focusing on state-of-the-art current (ALMA) and proposed (ngVLA) facilities.

Surfaces: The surfaces of moons preserve evidence of their thermal and geological histories, as well as exogenic modification processes. Geological activity such as Io's volcanism and Enceladus' polar plumes are associated with enhanced thermal emission and altered thermophysical properties due to fresh deposits, while potential upwelling in Europa's chaos regions may produce thermal signatures that remain to be detected. Thermal observations can also identify the primary source(s) of exogenic surface modification, a poorly understood area for the jovian satellites, which orbit within a complex system of neutral and ionized material.

At $\mathrm{mm}$ and $\mathrm{cm}$ wavelengths, the thermal continuum from 30-150 K (sub-)surfaces is readily detected. The intensity and polarization of the emission constrain thermophysical surface properties such as the thermal conductivity, dielectric constant, and emissivity, which in turn shed light on the surface's density, composition, and roughness. At these wavelengths, thermal emission arises from centimeters to meters within the subsurface, providing quantitative information on temperature and material properties as a function of depth. Surface roughness at the scale of a wavelength is best constrained by the polarization of the emission, and is of particular interest for targets where landed missions are being considered. Observations at a spatial resolution of $\sim 50 \mathrm{~km}$ or better will begin to resolve individual geological terrains, allowing for a comparison of the material properties between terrains on the same satellite.

Atmospheres: The radio and sub-millimeter wavebands also provide sensitive access to rotational transitions of dipolar, gas-phase molecules in planetary atmospheres and exospheres, enabling detailed studies of atmospheric physics, dynamics, and chemistry that can also be linked to surface and subsurface processes. The ability to directly detect and map emission from potential reservoir volatiles such as $\mathrm{H}_{2} \mathrm{O}, \mathrm{NH}_{3}, \mathrm{HCN}, \mathrm{H}_{2} \mathrm{CO}, \mathrm{SO}_{2}, \mathrm{H}_{2} \mathrm{~S}, \mathrm{NaCl}, \mathrm{KCl}$, and $\mathrm{CH}_{3} \mathrm{OH}$ presents a powerful and currently underutilized method for probing the chemistry of subsurface liquid reservoirs of Solar System bodies, which otherwise remain inaccessible to remote sensing. The cutting-edge ground-based facility ALMA has provided fundamental insights into the atmospheric properties of Io, Titan, Triton and Pluto, and promises to advance our understanding of the little-studied exospheres of ocean worlds such as Europa, Ganymede, Callisto and Enceladus during the next decade (Palmer et al., 2017; Gurwell et al., 2019; de Pater et al., 2020). Through studies of ocean world plumes, remote habitability studies are becoming possible for the first time, from the ground, while observations of volcanic activity provide information on geophysical processes and the properties of source reservoirs. However, the weak molecular 
emission from low-density atmospheres stretches the limits of our current observing capabilities, requiring investment in the next generation of instruments to continue to advance our understanding of tenuous satellite atmospheres.

With the current convergence of missions to the Solar System's satellites, now is the time to optimize our ground-based facilities and observing capabilities to support and augment the science return from in situ exploration. In order to provide the necessary global and temporal context for highly-targeted measurements to be made by missions such as Europa Clipper, Dragonfly and JUICE, we recommend continued, dedicated investment in infrastructure for ground-based radio and radar interferometry of satellite surfaces and atmospheres. Ground-based radio $\mathrm{mm}-\mathrm{cm}$ interferometry also fills the gap in depth coverage between infrared thermal instruments and ground-penetrating radar; detects and maps atmospheric species, placing the more targeted spacecraft observations in global context; and is even sensitive to minor species such as isotopologues that planned spacecraft mass spectrometers lack the mass resolution to isolate.

\subsection{Current and future facilities}

Radio interferometry is capable of achieving tens to even hundreds of resolution elements across targets such as the galilean satellites from Earth, but the combined baseline length and sensitivity have not been sufficient for such experiments until the Atacama Large (sub)Millimeter Array (ALMA). ALMA has enabled detailed thermal and atmospheric observations of satellites across the Solar System, as described throughout the text. The next-generation VLA (ngVLA) is a proposed radio interferometer (see McKinnon et al. (2020)) that would extend the high sensitivity and spatial resolution of ALMA to longer wavelengths (2.5 $\mathrm{mm}-25 \mathrm{~cm}$ ), probing much deeper in satellite surfaces and sensing different molecular species and excitation temperatures at a spatial resolution that is a factor of 5-10 higher than a 10-meter infrared telescope with adaptive optics, or the Hubble Space Telescope in the optical, and corresponding to $\sim 18 \mathrm{~km}$ in the jovian system and $\sim 35 \mathrm{~km}$ at Saturn. Its large collecting area and high bandwidth will enable sub-Kelvin sensitivity, providing roughly the same sensitivity at $1 \mathrm{~cm}$ as ALMA does at $1 \mathrm{~mm}$.

\subsubsection{Bistatic Interferometric Radar}

Planetary radar is a related, powerful technique to probe the characteristics of bodies in the solar system, and discovered for example the extremely high radar reflectivity and polarization ratio of the icy satellites (Galilean satellites - Ostro et al. (1992); Saturnian satellites - Black et al. (2007)). Unfortunately, the giant planet satellites are too far away, and have observing geometry that prevents traditional delay-doppler imaging using monostatic radar (transmitting and receiving with the same telescope) or bistatic single-dish radar (transmitting from one antenna and receiving from another). While radar images of Ganymede and Callisto have been produced with monostatic and bistatic interferometric radar (transmitting from one antenna and receiving with an array of antennas), the fidelity and resolution are limited (Harcke, 2005).

Radar imaging of the giant planet satellites would be enabled by the installation of a 500 $\mathrm{kW}$ 1-cm transmitter on the Green Bank Telescope (GBT), combined with the ngVLA as the 


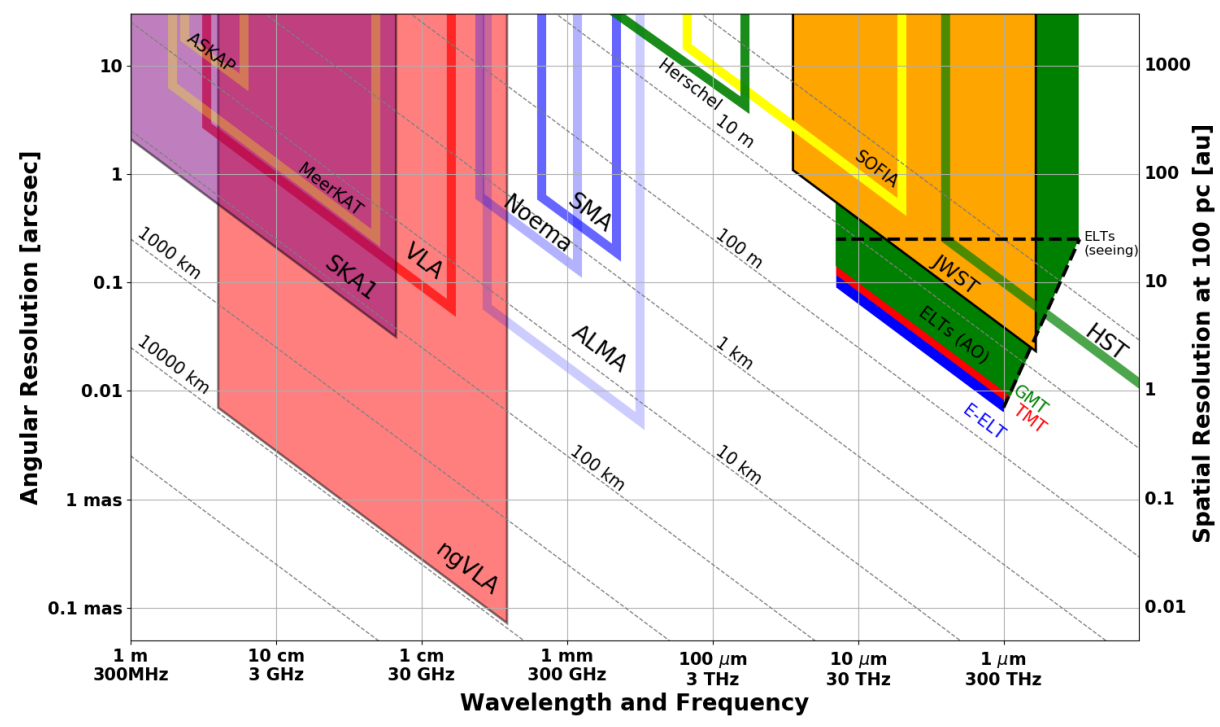

Figure 1: The ngVLA and ALMA in the context of the current \& nextgen observatory landscape. 1 mas is approximately $3.5 \mathrm{~km}$ in the Jupiter system and $7 \mathrm{~km}$ in the Saturn system.

receiving end of a bistatic interferometric radar. Such a radar has been proposed to the Astro2020 decadal panel (Bonsall et al., 2019). With this combination of instruments, radar images of the galilean satellites could be made with 50 pixels across the target (similar to the resolution on Mars using the Goldstone-VLA radar), 10 pixels across Titan and a few across Rhea. Several smaller satellites could be readily detected, including the eight non-Galilean Jovian moons with diameter $>35 \mathrm{~km}$, all 6 of the other large Saturnian moons, and the 4 largest moons of Uranus. These experiments would undoubtedly yield incredible results, just as the previous, more limited experiments have in the past.

\subsubsection{Requirements}

Future facilities need to provide the following capabilities to enable high-impact Solar System science: (1) non-sidereal tracking at a minimum rate of 1 "/s, as well as the logistical ability to schedule with fine time precision to e.g. follow a satellite in an eclipse (30-60 minute window); (2) ToO capabilities to follow up on a transient event detection such as a volcanic eruption or water plume outburst. (3) An angular resolution of 0.05" or better to resolve geological terrains. (4) Broad frequency coverage $(\lesssim 1-116 \mathrm{GHz}$ for the case of ngVLA) would enable the broadest range of science. Even higher frequencies, up to hundreds of $\mathrm{GHz}$, are optimal for molecular spectroscopy. Finally, measurements of (5) all 4 Stokes parameters are required.

\section{Selected science cases}

\subsection{Titan}

Titan's surface conditions are near the triple point of methane, like water on Earth. Cassini data have revealed the presence of lakes and seas (Stofan et al., 2007), a surface modified by aeolian and fluvial processes, and probable methane rain (Turtle et al., 2011; Dhingra et al., 
2019). This dynamic system is driven by interactions between the troposphere and (sub)surface, yet both of these regions are poorly sampled, which is a limiting factor on the development of climate models (Tokano, 2014).

Continuum emission at mm-cm wavelengths originates from the (sub)surface and troposphere. With a spatial resolution of $225 \mathrm{~km}$ achieved at a sensitivity of $0.3 \mathrm{~K}$ in just a few hours, the ngVLA 1-cm band will allow high precision surface brightness temperature maps for exploration of limb darkening, localized emissivity, and latitudinal temperature gradients. The 2-cm band will allow a direct, high-resolution comparison to the Cassini RADAR dataset, following up on detections of large scale variations in brightness that were attributed to variations in surface roughness (enhancing scattering) and in absorptivity (dielectric constant) of the surface materials. Since the end of the Cassini mission, continuum observations using ground-based radio arrays present our only means of making well-resolved thermal observations of active satellites in the saturnian system, and of extending Cassini's rich scientific legacy of Titan atmospheric studies by continuing observation through a full Titan year. The global perspective provided by ALMA and the ngVLA would also place the more localized studies that will be performed by Dragonfly in context, and will complement the mission through the sensitivity to large-scale seasonal changes on Titan's surface.

At the higher frequencies sensitive to the atmosphere, ALMA spectroscopy has enabled detections of new organic molecules in Titan's atmosphere, including species of possible astrobiological relevance (Palmer et al., 2017). The ability to form biologically relevant molecules including amino acids and DNA nucleobases has already been demonstrated in the laboratory under Titan-like conditions (Hörst et al., 2012), but Titan's full organic inventory is still not observationally well-constrained. The 1-3 mm waveband covered by ALMA and the ngVLA is well suited for the detection of complex organics (e.g., $\mathrm{HCN}, \mathrm{HC}_{5} \mathrm{~N}, \mathrm{HC}_{7} \mathrm{~N}$ ), that could make their way to the surface for participation in pre-biotic chemical synthesis. The improved sensitivity offered by a next-generation radio interferometer will enable sensitive searches for biomolecules such as adenine (the most plausible nucleobase for detection on Titan), in addition to nitrogen-bearing heterocycles (the building blocks of nucleobases), and amino acid precursors such as methylamine $\left(\mathrm{CH}_{3} \mathrm{NH}_{2}\right)$ and aminoacetonitrile $\left(\mathrm{NH}_{2} \mathrm{CH}_{2} \mathrm{CN}\right)$. The greatest sensitivity will be achieved via broadband, multi-line searches, combined with detailed spectral modeling, incorporating accurate laboratory spectroscopy of the species of interest.

\subsection{Io}

The composition, temperature structure and dynamics of Io's atmosphere remain poorly understood, and investigation into its nature and variability are limited by the difficulty of detecting the tenuous atmospheric signatures above the bright surface reflectance at UV through NIR wavelengths. As a result, Io's bulk $\mathrm{SO}_{2}$ atmosphere, as well as many other gaseous species, were first detected at millimeter wavelengths (Lellouch et al., 1990; Moullet et al., 2013); the gasses detected to date at these wavelengths include $\mathrm{SO}_{2},{ }^{34} \mathrm{SO}_{2}, \mathrm{SO}, \mathrm{NaCl}$, and $\mathrm{KCl}$ (Moullet et al., 2010, 2013; de Pater et al., 2020) - see Figure 2. The spatial distribution of the alkalis confirms that these species are localized to distinct regions (Moullet et al., 2015), several of which correspond to known volcanic sources, though $\mathrm{SO}_{2}$ emission is notably absent, perhaps suggesting variations in magma composition (de Pater et al., 2020).

The lower frequencies sensed by the ngVLA would enable searches for new species, as 

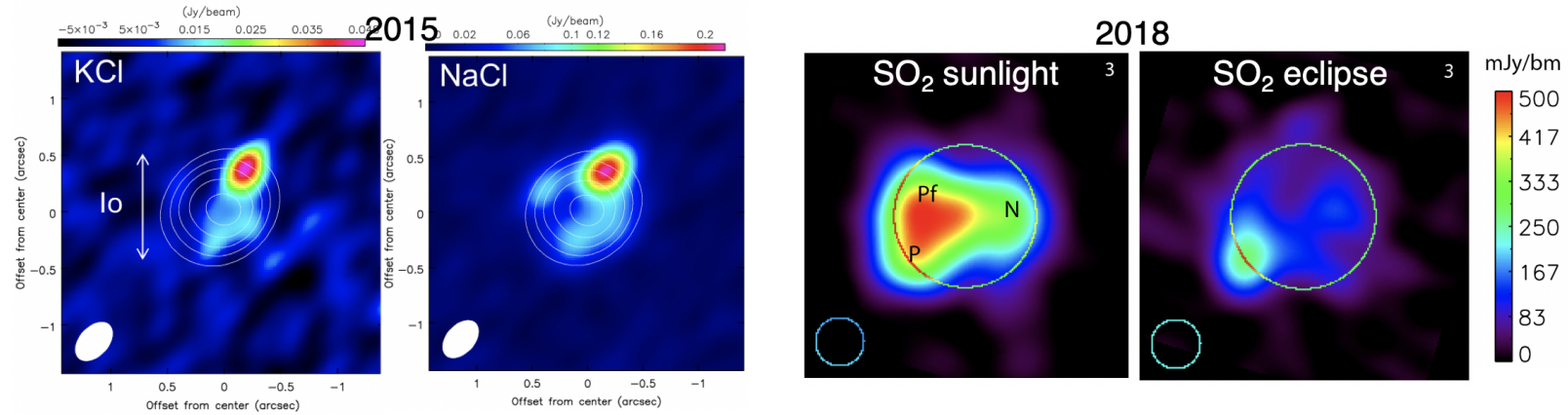

Figure 2: Spatial inhomogeneities in Io's $\mathrm{SO}_{2}$ and alkali gasses are easily seen in spatially-resolved ALMA maps of Io in both sunlight and eclipse (A. Moullet; de Pater et al. (2020)). The ngVLA will achieve a $>10 x$ higher spatial resolution at Io than these data, possibly even resolving the plumes themselves.

well as the mapping of lines from known species (e.g. $\mathrm{SO}, \mathrm{SO}_{2}$ ) over a wider range of excitation temperatures, improving the interpretation of existing datasets through providing a better characterization of Io's atmospheric temperature structure. Io's atmosphere also varies spatially and temporally following the distribution of surface frosts, the changing solar insolation, the pattern of particle bombardment, and the locations of active plumes. The unmatched sensitivity of the ngVLA would allow clear detections of abundant species in short on-source times, permitting studies of volcanic variability and diurnal atmospheric cycles, which both are largely unexplored arenas. Data at the ngVLA's high spatial resolution would reveal spatial correlations with surface properties, constrain atmospheric temperature (a big unknown at present, with values ranging from $<140$ to over $500 \mathrm{~K}$ ), resolve volcanic plumes, and would provide a window into atmospheric dynamics through the mapping of line-of-sight wind speeds from Doppler shift measurements.

Io also represents an important test case for understanding tidal heating. A major outstanding question regarding Io's heat flow is how the heat is transported from the interior to surface, given that the volcanoes themselves can only account for half (Veeder et al., 2015). The Io Volcano Observer (IVO) mission currently under study for NASA's Discovery program would map the thermal emission from Io's surface away from volcanoes in the infrared. The constraints these data will place on Io's directly conducted heat flow would be tightened by a better characterization of the material properties and heat flow through the subsurface that $\mathrm{mm}-\mathrm{cm}$ observations would provide. A spatial resolution of $100 \mathrm{~km}$ or better is required to distinguish active lava flows from passive surface regions. $\mathrm{Mm}-\mathrm{cm}$ data also sense beneath superficial deposits or even thin flows, and would provide information on lava flow texture at roughly the scale of a wavelength, which constrains viscosity and flow rate; recent data from ALMA indicate that a denser, higher thermal inertia layer underlays the fluffier surface material at a depth of a few cm (de Pater et al., 2020).

\subsection{The Icy Galilean Satellites}

On satellites with subsurface oceans, regions where warmer subsurface material is transported to the near-surface could manifest as 'hot spots' detectable in thermal emission. 

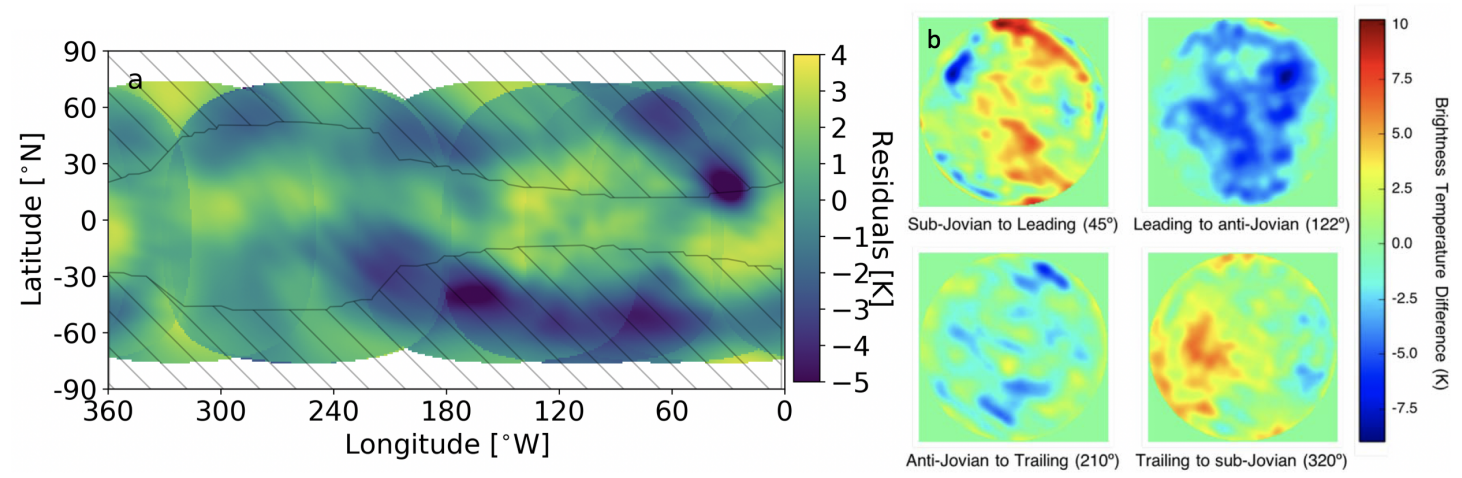

Figure 3: ALMA can reveal temperature variations of a few $\mathrm{K}$ on spatial scales of a few hundred $\mathrm{km}$ at the galilean satellites. Figure shows temperature residuals from best fit thermal models to ALMA observations of (a) Ganymede, with the region where magnetic field lines contact the surface highlighted, and (b) Europa. Both show localized regions where thermal properties differ from best fit values (Trumbo et al., 2018; de Kleer et al., 2020). The ngVLA would sense deeper in the surface, at $>10 x$ higher spatial resolution than the data shown here.

Spatially-resolved observations with the IRAM interferometer and ALMA have provided millimeter-wavelength brightness temperature maps of the galilean satellites at hundreds of $\mathrm{km}$ resolution, some of which are shown in Figure 3 (Moullet et al., 2008; Trumbo et al., 2017; de Kleer et al., 2018; Trumbo et al., 2018). Such data have been used to demonstrate the distinctive thermal properties of impact crater ejecta, and to confirm that a nightside hot spot on Europa detected near Pwyll Crater by Galileo is in fact an area of high thermal inertia rather than enhanced heat flow (Trumbo et al., 2017).

Disentangling the causes of thermal anomalies on these satellites is vital to our understanding of the endogenic heat flow of tidally-heated ocean worlds, as well as the relationships between their surface thermal properties and external processes such as particle irradiation, sputtering, and electron sintering. The ngVLA, which would sense deeper in the surface at the high spatial resolution needed to isolate geological terrains, would robustly constrain the density and temperature profile down to meters depth and hence isolate an endogenic heat flow component if present, as well as providing temperature profiles to constrain calculations of the diffusion rate of materials between a subsurface ocean and the surface, an important parameter in potential habitability. Ground-based measurements at mm-cm wavelengths fill in a key gap in wavelength (and hence depth) coverage between Europa Clipper's thermal imager and ground-penetrating radar, enabling the fitting of self-consistent models to multi-wavelength datasets and the reconstruction of subsurface temperature and material properties profiles.

Localized plumes are present on Enceladus, Io, Triton, and perhaps Europa. Enceladus' plume is dominated by water, but molecules such as ammonia, methanol, formaldehyde, and even more complex organics have been detected (Postberg et al., 2018). Millimeter wavelengths are uniquely sensitive to many of these species, and spectral line observations present a potential avenue for continuing to track the plume's variability, and for detecting and measuring molecular abundances. 


\section{Summary}

The solar system's satellites span a wide range of properties and thermal histories. A subset of these worlds host geological activity, subsurface oceans, and/or complex atmospheric chemistry and are of great interest from both geophysical and astrobiological perspectives. Radio frequencies are uniquely sensitive to thermal emission from cold satellite (sub)surfaces and to trace molecular species. Current and proposed interferometry arrays at millimeter-centimeter wavelengths can now achieve a comparable spatial resolution at thermal wavelengths as can be achieved in reflection with the best ground-based infrared-optical observatories. These capabilities have been demonstrated with ALMA, and can be extended to deeper into satellite subsurfaces and to additional molecular transitions with the construction of the ngVLA, providing a more robust link between surface and atmospheric properties (including plumes) and interior processes. In combination with the ngVLA, the construction of a $500 \mathrm{~kW} \mathrm{1-cm} \mathrm{transmitter} \mathrm{at} \mathrm{the}$ GBT would also enable interferometric radar mapping of these worlds. We urge continued investment in ground-based radio interferometric capabilities, in particular the continued development and operation of ALMA and the construction of the ngVLA; these facilities have the potential to profoundly impact our understanding of satellite surfaces and atmospheres, and will enhance the return from numerous planned spacecraft missions.

The authors acknowledge ideas generated in the Next-Generation Planetary Radar workshop organized by the W.M. Keck Institute for Space Studies.

\section{References}

Anderson et al. 2014. Icarus, 243, 129.

Black et al. 2007. Icarus 191, 702.

Bonsall et al. 2019. BAAS 51, 208.

Butler 2012. ALMA Memo 594.

de Kleer et al. 2018. LPSC 2083, 2567.

de Kleer et al. in prep.

de Pater et al. 1984. Icarus 57, 93.

de Pater et al. 2018. arXiv:1810.08521

de Pater et al. 2020 in review

Dhingra et al. 2019. GRL, 46.

Fulchignoni, et al. 2005. Nature 438, 785.

Gary et al. 1965, ApJS 12, 239.

Gurwell et al. 2019. EPSC 2019-806.

Harcke 2005. PhDT, Stanford Uni.

Hörst et al. 2012. Astrobio 12, 809.

Hörst 2017, JGR Planets 122, 432.

Ingersoll, Ewald 2017. Icarus 282, 260.

Jennings et al. 2016. ApJ 816, L17.

Le Gall et al. 2016. JGR Planets 121, 233.

Lellouch et al. 1990. Nature 346, 639.

Lindal et al. 1983. Icarus 53, 348.
McKinnon et al. 2020. Astro2020 APC White Paper.

Moullet et al. 2008. A\&A 482, 279.

Moullet et al. 2010. Icarus 208, 353.

Moullet et al. 2013. ApJ 776:32.

Moullet et al. 2015. AAS DPS \#47, 311.31

Muhleman, Berge, 1991. Icarus 92, 263.

Niemann et al. 2010, JGR Planets 115, E12006.

Nowling, Moullet 2015. AAS \#225, 137.11.

Ostro et al. 1992. JGR 97, 18227.

Palmer, et al. 2017. Science Advances 3, 7.

Postberg et al. 2018. Nature 558, 564.

Schinder et al. 2012. Icarus 221, 1020.

Stofan, et al. 2007. Nature 445, 61.

Tokano 2014, Icarus 231, 1.

Trumbo et al. 2017. AJ 154:148.

Trumbo et al. 2018. AJ 156:161.

Turtle et al. 2011, Science 331, 1414.

Ulich et al. 1984. Icarus 60, 590.

Veeder et al. 2015. Icarus 245, 379. 